\title{
Cardio-Respiratory Characterization of the Autonomic Balance
}

\author{
Leila Mirmohamadsadeghi ${ }^{1}$, Nicolas Bourdillon ${ }^{2}$, Grégoire P. Millet ${ }^{2}$, Jean-Marc Vesin ${ }^{1}$ \\ ${ }^{1}$ Applied Signal Processing Group, Swiss Federal Institute of Technology, Lausanne, Switzerland \\ ${ }^{2}$ ISSUL Institute of Sport Sciences, University of Lausanne, Switzerland
}

\begin{abstract}
The autonomic balance is often measured using the low frequency $(L F)$ and high frequency $(H F)$ powers of the heart beat-to-beat intervals. However, these indices do not adequately integrate the influence of respiration and have been widely criticized. We studied the autonomic balance with measures from the heart beat-to-beat intervals taking into account the respiratory activity. Using cardiac and respiratory data acquired simultaneously from healthy volunteers in supine and orthostatic positions, we found that the investigated measures convey changes in the autonomic balance in a physiologically meaningful manner in contrast to the classic LF and HF indices.
\end{abstract}

\section{Introduction}

The cardiac autonomic balance, describing the activations of the sympathetic and parasympathetic branches of the autonomic nervous system (ANS), is often noninvasively measured through heart rate variability (HRV). In HRV analysis, which is the analysis of the heart inter-beat intervals variations, the powers in the low frequency (LF) band of the inter-beat intervals, between 0.04 and $0.15 \mathrm{~Hz}$, believed to be influenced by both the sympathetic and parasympathetic branches of the ANS, and high frequency (HF) band between 0.15 and $0.4 \mathrm{~Hz}$, thought to be influenced by the parasympathetic branch of the ANS, are measured as markers [1]. Furthermore, the ratio LF/HF of the powers is sometimes used as a marker of sympathetic activation [2,3]. However, the LF-HF partitioning and the use of the LF/HF marker are widely contested [3-6], in part because there is evidence against a clear linear separability of the activations of the two branches of the ANS, but also due to the influence of the respiration on the HRV [3-5]. The respiration may occur in the LF or the HF band, or jump from one to the other within a given experimental protocol, and since it has a large influence on the power of the inter-beat intervals, it cannot be ignored when performing HRV analysis. Some researchers have proposed to analyze the respiratory and non-respiratory HRV [4], while others propose to center the HF band on the respiratory frequency and to adjust the LF boundary accordingly [6].

In addition to HRV analysis, the synchronization of the phase lag between the respiration and the respiratory component of the inter-beat intervals, referred to as the respiratory sinus arrhythmia (RSA), has been proposed as a potential autonomic index [7], taking root in ANS-mediated synchronization aspects of the cardio-respiratory system.

In the present study, we aim to apply and compare respiration-corrected HRV analysis techniques in addition to investigating measures engulfing synchronization aspects of the respiration influence on the HRV. These measures are compared for the supine and orthostatic body positions, assumed to elicit parasympathetic and sympathetic dominance, respectively. We used electrocardiogram (ECG) and respiration data from healthy subjects. By least-squares identification, the RSA was extracted from the inter-beat intervals time series of the ECG. The RSA power (respiratory) and the non-respiratory power of the inter-beat intervals were measured. The variability and the synchronization of the relative phase lag of the RSA and the respiration were also analyzed. The respirationcorrected LF and HF powers and their ratio, and the classic LF and HF powers and their ratio were measured as well for comparison.

\section{Methods}

\subsection{Data}

The data used in this study were the ECG and airflow (spirometer) acquired from 21 healthy volunteers (16 men, age $34 \pm 8$ years, weight $78 \pm 17 \mathrm{~kg}$, height $178 \pm 9 \mathrm{~cm}$ ) while they lay supine for 8 minutes and then stood in the orthostatic position for 7 minutes. All study procedures were in accordance with the Declaration of Helsinki, and the study was approved by the local ethical committee (\#2016-00308). The subjects gave informed oral consent prior to participation. The data were acquired with a sampling frequency of $1000 \mathrm{~Hz}$. 
Table 1. Summary of the measured parameters.

\begin{tabular}{|c|c|c|}
\hline Signal(s) & Parameter & Definition \\
\hline \multirow{5}{*}{ R-R intervals } & LF & power in the $0.04-0.15 \mathrm{~Hz}$ band \\
\hline & $\mathrm{nLF}$ & normalized LF power \\
\hline & $\mathrm{HF}$ & power in the $0.15-0.4 \mathrm{~Hz}$ band \\
\hline & $\mathrm{nHF}$ & normalized HF power \\
\hline & $\mathrm{LF} / \mathrm{HF}$ & ratio of LF to HF powers \\
\hline \multirow{5}{*}{$\mathrm{R}-\mathrm{R}$ intervals \& respiration } & cLF & LF upper bound corrected (with respect to the respiratory frequency) power \\
\hline & ncLF & normalized cLF power \\
\hline & $\mathrm{cHF}$ & HF lower bound corrected (with respect to the respiratory frequency) power \\
\hline & $\mathrm{ncHF}$ & normalized cHF power \\
\hline & $\mathrm{cLF} / \mathrm{cHF}$ & ratio of cLF to cHF powers \\
\hline \multirow{2}{*}{ RSA } & RSA & RSA power \\
\hline & NRE & non-respiratory power (R-R - RSA) \\
\hline \multirow{3}{*}{ RSA \& respiration } & VPL & variation of the phase lag between the RSA and the respiration \\
\hline & SPL & synchronization of the phase lag between the RSA and the respiration \\
\hline & $\mathrm{AR}$ & amplitude of the RSA to the respiration \\
\hline
\end{tabular}

\subsection{Processing}

The ECG R peaks were extracted with a classic extrema detection method and manually inspected for artifacts. The R-R intervals and the reference respiration were re-sampled at $4 \mathrm{~Hz}$ using cubic spline interpolation.

The RSA was extracted from the R-R intervals as follows: first both the respiration signal and the R-R intervals were filtered with a local band-pass filter centered on the instantaneous respiratory frequency, which was itself computed with the notch filter bank method [8]. This step yields a rough estimate of the respiratory component of the $\mathrm{R}-\mathrm{R}$ intervals, the RSA. The refined RSA was estimated with least-squares identification from the filtered R-R intervals by using the filtered respiration signal as reference. This estimate was further high-pass filtered with a Butterworth filter above $0.06 \mathrm{~Hz}$ to eliminate drift. The instantaneous frequency of the RSA was measured as well and compared to that of the respiration in order to ensure that the RSA did contain the respiratory-related information. The following parameters were measured using the power spectral density (PSD) of the RSA, computed with the Welch method in 30-second long sliding windows with 50\% overlap:

(a) The RSA power.

(b) The non-respiratory power measured from the R-R intervals after the removal of the RSA, denoted by NRE.

The following parameters were computed jointly from the filtered respiration and the RSA, describing their relationship:

(a) The variation of the phase lag between the two, denoted by VPL, computed as the standard deviation of the slope of the phase lag similarly to [9].

(b) The synchronization of the phase lag as described in [7], denoted by SPL.

(c) The ratio of the RSA instantaneous amplitude to that of the respiration, denoted by AR, thought to carry information on the relative importance of the RSA. The amplitudes were assessed by using the mean envelopes (mean of lower and upper envelopes), computed by interpolating (cubic) the locations of the local extrema of the signals.

For comparison, the following parameters were measured from the R-R interval series PSD (computed with the Welch method in 30-second long sliding windows with $50 \%$ overlap):

(a) The LF power and the normalized LF power, denoted by nLF.

(b) The HF power and the normalized HF power, denoted by nHF.

(c) The LF/HF index.

The following parameters were measured from the R-R intervals PSD by setting the LF-HF boundary according to the respiration frequency as $\min \left(f_{\text {resp }}-\delta f, 0.15\right)$, where two values of 0.05 and $0.1 \mathrm{~Hz}$ were investigated for $\delta f$ :

(a) The corrected LF power, denoted by cLF, and the normalized cLF, denoted by ncLF.

(b) The corrected $\mathrm{HF}$ power, denoted by $\mathrm{cHF}$, and the normalized cHF, denoted by ncHF.

(c) The cLF/cHF index.

Table 1 summarizes the measured parameters. For each parameter, the mean values pertaining to the two positions were compared over all subjects with the Wilcoxon signed rank test, which does not assume prior information about the distribution of the data. 
(a) Supine

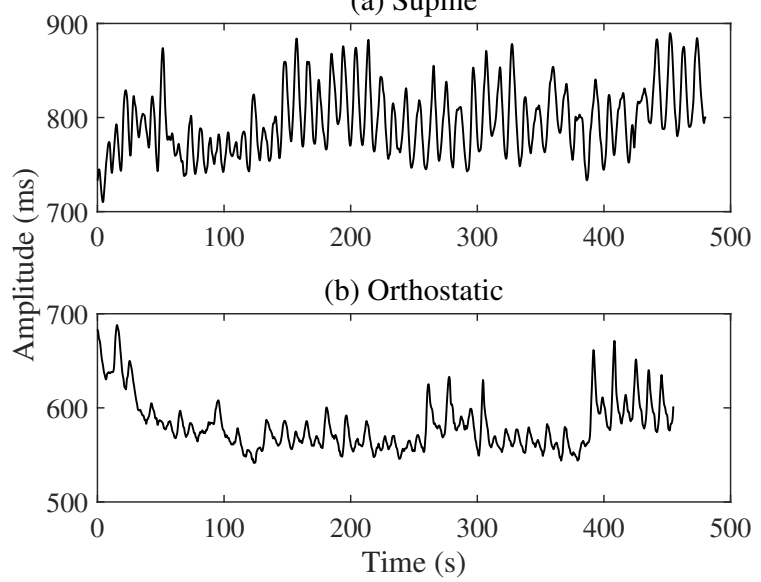

Figure 1. The R-R intervals for one subject in the (a) supine and (b) orthostatic positions.

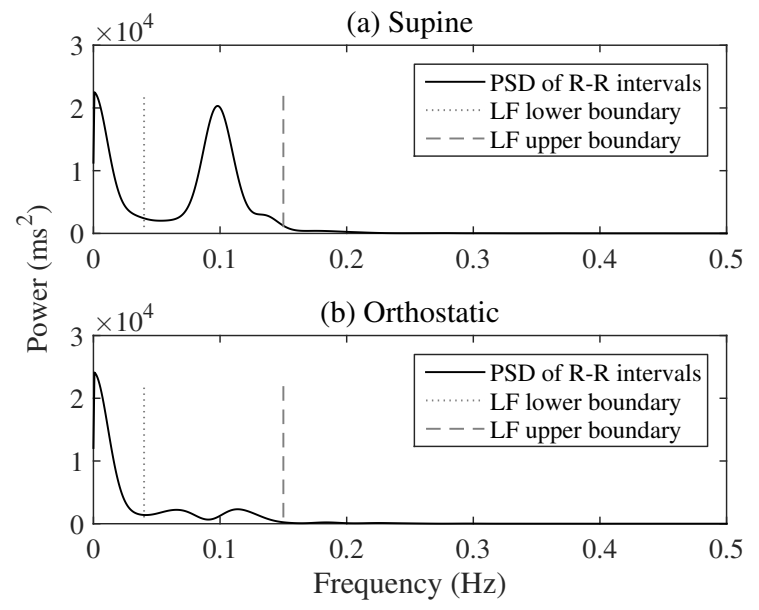

Figure 2. The PSD of the R-R intervals in Figure 1 in the (a) supine and (b) orthostatic positions. In both cases, the respiration occured in the LF band, rendering the usual LF-HF separation meaningless.

\section{Results}

There were no significant differences between the instantaneous frequencies of the RSA and the respiration, meaning that the RSA extraction was correct for all subjects. The respiration rates of 12 subjects $(57 \%)$ were below the LF upper boundary of $0.15 \mathrm{~Hz}$. For 6 of these subjects (28\% of total), it was close to the frequency of the baroreflex activity $(0.1 \mathrm{~Hz})$.

Figure 1 shows the R-R intervals for one subject for the supine and orthostatic positions. Figure 2 illustrates the PSD of the R-R intervals from Figure 1. Visually, the respiratory influence on the R-R intervals, seen in the main oscillation, is more prominent in the supine position.
Table 2. Comparison of the HRV parameters between the supine and orthostatic positions over all subjects.

* Supine $>$ Orthostatic with $p<0.05$,

${ }^{\dagger}$ Supine $<$ Orthostatic with $p<0.05$, otherwise non-significant.

\begin{tabular}{lcc}
\hline \hline Parameter & Supine & Orthostatic \\
\hline $\mathrm{LF}\left(m s^{2}\right)$ & $2698.17 \pm 2510.31$ & $4270.06 \pm 6053.12$ \\
$\mathrm{nLF}$ & $0.42 \pm 0.19^{\dagger}$ & $0.57 \pm 0.19$ \\
$\mathrm{HF}\left(m s^{2}\right)$ & $2949.72 \pm 5045.81^{*}$ & $638.03 \pm 979.84$ \\
$\mathrm{nHF}$ & $0.36 \pm 0.19^{*}$ & $0.10 \pm 0.08$ \\
$\mathrm{LF} / \mathrm{HF}$ & $3.30 \pm 5.50^{\dagger}$ & $12.53 \pm 10.55$ \\
\hline $\mathrm{cLF}\left(m s^{2}\right)$ & $921.21 \pm 1081.89$ & $1019.70 \pm 1518.37$ \\
$\mathrm{ncLF}$ & $0.17 \pm 0.12^{\dagger}$ & $0.29 \pm 0.20$ \\
$\mathrm{cHF}\left(m s^{2}\right)$ & $4726.68 \pm 6423.29^{*}$ & $3919.15 \pm 6690.86$ \\
$\mathrm{ncHF}$ & $0.62 \pm 0.17^{*}$ & $0.37 \pm 0.31$ \\
$\mathrm{cLF} / \mathrm{cHF}$ & $0.44 \pm 0.54^{\dagger}$ & $4.39 \pm 6.79$ \\
\hline $\mathrm{RSA}\left(m s^{2}\right)$ & $1770.92 \pm 1689.72^{*}$ & $1757.17 \pm 3255.50$ \\
$\mathrm{NRE}\left(m s^{2}\right)$ & $535.65 \pm 646.59^{*}$ & $433.12 \pm 672.65$ \\
\hline $\mathrm{VPL}(\mathrm{rad})$ & $0.08 \pm 0.04^{\dagger}$ & $0.26 \pm 0.23$ \\
$\mathrm{SPL}$ & $0.98 \pm 0.01^{*}$ & $0.90 \pm 0.13$ \\
$\mathrm{AR}$ & $0.21 \pm 0.14^{*}$ & $0.12 \pm 0.15$ \\
\hline \hline
\end{tabular}

In both graphs of Figure 2, a peak can be seen around $0.1-0.11 \mathrm{~Hz}$ because the respiratory rate of this subject for both recordings was $0.11 \mathrm{~Hz}$, which is close to the baroreflex frequency. In this case, the HF power does not represent respiratory activity at all, and the LF power contains both respiratory and baroreflex-related power.

Table 2 reports the mean values of the parameters described in Section 2.2 over all subjects. For the computation of cLF, ncLF, $\mathrm{cHF}$ and $\mathrm{ncHF}, \delta f$ was set to $0.05 \mathrm{~Hz}$ because with $\delta f=0.1 \mathrm{~Hz}$, these indices could only be computed for 16 subjects, as the others had respiration rates too close to the LF lower boundary. It can be seen that despite $57 \%$ of the subjects having respiratory rates outside the HF band, the HF and nHF powers were significantly higher in the supine position that in the orthostatic. The LF power showed a non-significant increase in the orthostatic position, while the nLF significantly increased. cLF, $\mathrm{ncLF}, \mathrm{cHF}$ and ncHF exhibited the same behavior as their classic counterparts, although the differences were less extreme. The RSA power, which in effect was sometimes in the HF and sometimes in the LF and sometimes overlapping both bands, was significantly higher in the supine position. The non-respiratory power, NRE, followed the same trend as the RSA power. The synchronization of the phase lag was significantly higher in the supine position and its variance was significantly lower. The amplitude ratio of RSA to the respiration amplitudes was significantly higher in the supine position. 


\section{Discussion and Conclusion}

The classic LF and HF power measures report significant differences between supine and orthostatic positions, despite respiration being in the HF band of the inter-beat intervals for less than half the subjects. This could mean that the non-respiratory influence on the R-R intervals is also affected by change in position. These measures should be used with caution.

The corrected cLF and cHF powers exhibited the same behavior as the classic LF and HF powers and the $\mathrm{CLF} / \mathrm{cHF}$ followed a behavior similar to that of the LF/HF index, however, the differences were less extreme. The cLF, ncLF and $\mathrm{cLF} / \mathrm{cHF}$ were higher in the orthostatic position, consistent with the increase in sympathetic activation in this position. The cHF and ncHF were higher in the supine position, for which the parasympathetic system dominates. The values of cLF were lower than those of the classic LF power, and those of the cHF were higher than the classic HF, accounting for the difference due to subjects whose respiration was below the LF upper boundary. It must be noted that the definitions of the corrected LF and HF bands were arbitrary and there is no unique accepted definition for these parameters. Furthermore, depending on this definition, for some subjects with low respiration rates, it was not possible to compute the cLF and cHF powers, which induces limitations in the use of these indices. These indices seem to be physiologically interpretable alternatives to their classic counterparts. However, they cannot be easily applied to a wide range of cases.

The RSA, defined as the respiratory influence on the $\mathrm{R}-\mathrm{R}$ intervals, identified by using the reference respiration waveform, seems stronger in the supine position. Indeed, the RSA power was larger (although not by much), variations in the phase lag between the respiration and the RSA were lower, and the relative importance of the RSA amplitude to the respiration was larger. Furthermore, since the synchronization of the phase lag was larger in the supine position, one can assume that the RSA and the respiration had a more stable relationship in the supine position. These physiologically interpretable measures approach the problem of the autonomic balance from a different perspective. Instead of attempting to separate sympathetic and parasympathetic influences, these measures report physiological changes resulting from shifts in the autonomic balance.

Future work will focus on the removal of the respiration influence from the R-R intervals prior to HRV analysis, even when no direct respiration reference signal is available.

\section{Acknowledgements}

This work was funded thanks to the Swiss NanoTera initiative, RTD project ObeSense.

\section{References}

[1] Task force of the European Society of Cardiology and the North American Society of Pacing and Electrophysiology. Heart rate variability standards of measurement, physiological interpretation, and clinical use. Eur Heart J 1996;17:354381.

[2] Pagani M, Lombardi F, Guzzetti S, Sandrone G, Rimoldi O, Malfatto G, Cerutti S, Malliani A. Power spectral density of heart rate variability as an index of sympatho-vagal interaction in normal and hypertensive subjects. Journal of hypertension Supplement official journal of the International Society of Hypertension 1984;2(3):S383-5.

[3] Billman GE. The LF/HF ratio does not accurately measure cardiac sympatho-vagal balance. Frontiers in Physiology 2013;4:26.

[4] Widjaja D, Vlemincx E, Van Huffel S. Multiscale principal component analysis to separate respiratory influences from the tachogram: Application to stress monitoring. In Computing in Cardiology (CinC). September 2012; 277-280.

[5] Sidorenko L, Kraemer JF, Wessel N. Standard heart rate variability spectral analysis: does it purely assess cardiac autonomic function?, 2016.

[6] Hernando A, Lázaro J, Gil E, Arza A, Garzón JM, LópezAntón R, de la Cámara C, Laguna P, Aguiló J, Bailón R. Inclusion of respiratory frequency information in heart rate variability analysis for stress assessment. IEEE J Biomed Health Inform July 2016;20(4):1016-1025.

[7] Niizeki K, Saitoh T. Incoherent oscillations of respiratory sinus arrhythmia during acute mental stress in humans. Am J Physiol Heart C 2012;302(1):H359-H367.

[8] Mirmohamadsadeghi L, Vesin JM. Real-time multi-signal frequency tracking with a bank of notch filters to estimate the respiratory rate from the ECG. Physiol Meas 2016; 37(9):1573-1587.

[9] Mirmohamadsadeghi L, Fallet S, Buttu A, Saugy J, Rupp T, Heinzer R, Vesin JM, Millet GP. Sleep apnea detection using features from the respiration and the ECG recorded with smart-shirts. In IEEE Biomedical Circuits and Systems Conference (BioCAS) Proceedings. 2014; 61-64.

Address for correspondence:

Leila Mirmohamadsadeghi

EPFL SCI STI JMV ELD 234 Station 11

1015 Lausanne, Switzerland 